\title{
Fluctuations between the BCS and BEC Limits in the System of Ultracold Alkali Atoms
}

\author{
T. Domański and A. Donabidowicz \\ Institute of Physics, M. Curie Skłodowska University \\ pl. M. Curie-Skłodowskiej 1, 20-031 Lublin, Poland
}

\begin{abstract}
We investigate the fluctuations induced by time-dependent interchange between the Bardeen-Cooper-Schrieffer and Bose-Einstein condensation regimes in the ultracold gas of fermion atoms. Such crossover can be triggered by varying the external magnetic field across the Feshbach resonance. Experimental realization is usually done via very fast switching which leads to the nonequilibrium effects. In this paper we focus on the ground state properties. In particular, we analyze time dependence of the wave function and consider fluctuations of the order parameters.
\end{abstract}

PACS numbers: 74.20.Mn, 03.75.Kk, 03.75.Ss

\section{Introduction}

Ultracold fermion atoms, such as ${ }^{40} \mathrm{~K}$ or ${ }^{6} \mathrm{Li}$, are recently used to achieve a new kind of superfluidity where effective interactions are tuned via the magnetic field. In two-component systems made of the fermion atoms in two (or more) different hyperfine states it is possible to produce the weakly bound molecules with binding energy almost three orders of magnitude smaller than the helium dimers (the weakest bound pairs in the solid state physics) [1]. Such small size molecules get formed in presence of the magnetic field $B<B_{0}$, where the characteristic Feshbach resonance value $B_{0}$ depends on specific atoms and their Zeeman configurations [2]. At ultralow temperatures $T \sim T_{\mathrm{F}}$ (for $10^{5}$ trapped atoms with concentration $10^{13} \mathrm{~cm}^{-3}$ the typical Fermi temperature is $T_{\mathrm{F}} \approx 100 \mathrm{nK}$ ) these weakly bound molecules have been observed to undergo the Bose-Einstein condensation (BEC) [3].

Above the Feshbach resonance molecules are no longer bound. However, the virtual exchange of atoms into molecules generates an effective scattering potential $V \propto \frac{1}{B_{0}-B}[4]$ which for $B>B_{0}$ is attractive. This mechanism leads again to pair formation but in the momentum space. Fermion pairs, like the Cooper pairs of the Bardeen-Cooper-Schrieffer (BCS) theory, are there considered to be large. First 
experimental evidence for the superfluidity on the BCS side has been reported independently by several labs in 2004 [5].

Varying the magnetic field across the Feshbach resonance one can thus switch between the different limits of pairing and superfluidity. Although the theoretical concept of BCS to BEC crossover has a long time history [6] in the case of ultracold gases it can be realized for the first time in a fully controllable way.

Experimentally, switching between the BEC and BCS regimes is often done in time intervals much shorter than intrinsic scale set by the Fermi velocity $v_{\mathrm{F}}$. Nonequilibrium effects play then essential role and their description goes beyond the conventional frame of time-dependent Ginzburg-Landau equation. The fast time dynamics has been recently studied in the literature analytically [7] and numerically [8]. Unfortunately, no clear consensus has been reached concerning the damping effects for small and large size fermion pairs. In this paper we address this issue by considering the sinusoidal sweep between the BCS and BEC regimes. We show that for sufficiently large frequencies there appear oscillations of the order parameters.

\section{Microscopic model for the BCS to BEC crossover}

Basic physics of the fermion atoms correlated via the Feshbach resonance can be described by a two-channel model [9]:

$$
\begin{aligned}
H(t) & =\sum_{\boldsymbol{k}, \sigma}\left(\varepsilon_{\boldsymbol{k}}^{\mathrm{F}}-\mu\right) c_{\boldsymbol{k} \sigma}^{\dagger} c_{\boldsymbol{k} \sigma}+\sum_{\boldsymbol{q}}\left[\varepsilon_{\boldsymbol{q}}^{\mathrm{B}}+2 \nu(t)-2 \mu\right] b_{\boldsymbol{q}}^{\dagger} b_{\boldsymbol{q}} \\
& +\frac{g}{\sqrt{N}} \sum_{\boldsymbol{k}, \boldsymbol{q}}\left(b_{\boldsymbol{q}}^{\dagger} c_{\boldsymbol{q}-\boldsymbol{k} \downarrow} c_{\boldsymbol{k} \uparrow}+c_{\boldsymbol{k} \uparrow}^{\dagger} c_{\boldsymbol{q}-\boldsymbol{k} \downarrow}^{\dagger} b_{\boldsymbol{q}}\right) .
\end{aligned}
$$

Similar model has been also studied [10] as a scenario for the high temperature superconductivity. In the present context (1) refers to the fermion atoms in two Zeeman levels which are symbolically labeled by $\sigma=\uparrow$ and $\downarrow$. Kinetic energy of the single atoms is $\varepsilon_{\boldsymbol{k}}^{\mathrm{F}}=\hbar^{2} \boldsymbol{k}^{2} / 2 m$ while the energy of weakly bound molecules $\varepsilon_{\boldsymbol{k}}^{\mathrm{B}}=$ $\hbar^{2} \boldsymbol{k}^{2} / 2(2 m)$ is detuned from the Feshbach resonance by parameter $2 \nu(t)$. Common chemical potential $\mu$ ensures conservation of the total particle number $n_{\text {tot }}=$ $\sum_{\boldsymbol{k}, \sigma} c_{\boldsymbol{k} \sigma}^{\dagger} c_{\boldsymbol{k} \sigma}+2 \sum_{\boldsymbol{q}} b_{\boldsymbol{q}}^{\dagger} b_{\boldsymbol{q}}$ and the resonant scattering responsible for correlations between atoms comes from the boson-fermion exchange $g[2,4]$.

In the ground state we can approximately neglect the finite momentum bosons in (1). In that case it is possible to show [11] that the problem reduces to the well studied (but nontrivial) Dicke toy model [12]. In order to interpolate between the BCS and BEC limits we impose the following ansatz for the wave function [13]:

$$
|\Phi\rangle=\mathcal{N} \exp \left(\mathcal{A} b_{0}^{\dagger}+\sum_{\boldsymbol{k}} \mathcal{B}_{\boldsymbol{k}} c_{\boldsymbol{k} \uparrow}^{\dagger} c_{-\boldsymbol{k} \downarrow}^{\dagger}\right)|\mathrm{vac}\rangle
$$

where $\mathcal{N}$ is a normalization factor. The other two parameters $\mathcal{A}, \mathcal{B}_{\boldsymbol{k}}$ are time-dependent quantities and can be determined from a variational procedure for the 
action $S[\Phi]=\int \mathrm{d} t\left\langle\Phi\left|\left(\mathrm{i} \partial_{t}-H\right)\right| \Phi\right\rangle$. Let us recall that time dependence enters (2) via the detuning $2 \nu(t)$ which affects the relative populations of boson molecules and fermion atoms.

From the functional derivatives

$$
\frac{\delta S[\Phi]}{\delta \mathcal{A}}=0, \quad \frac{\delta S[\Phi]}{\delta \mathcal{B}_{k}}=0
$$

we derive the differential equations which determine the time dependence of $\mathcal{A}(t)$ and $\mathcal{B}_{\boldsymbol{k}}(t)$. Physical meaning of such parameters reads

$$
\mathcal{A}=\left\langle b_{\mathbf{0}}\right\rangle, \quad \mathcal{B}_{\boldsymbol{k}}=\frac{1-\left\langle\sigma_{\boldsymbol{k}}^{z}\right\rangle}{2\left\langle\sigma_{\boldsymbol{k}}^{-}\right\rangle} .
$$

We introduced here a convenient pseudospin notation [14] for the fermion pair operators $c_{-\boldsymbol{k} \downarrow} c_{\boldsymbol{k} \uparrow} \equiv \sigma_{\boldsymbol{k}}^{+}, c_{\boldsymbol{k} \uparrow}^{\dagger} c_{-\boldsymbol{k} \downarrow}^{\dagger} \equiv \sigma_{\boldsymbol{k}}^{-}$and for the particle number operator $c_{\boldsymbol{k} \uparrow}^{\dagger} c_{\boldsymbol{k} \uparrow}+c_{\boldsymbol{k} \downarrow}^{\dagger} c_{\boldsymbol{k} \downarrow} \equiv 1-\sigma_{\boldsymbol{k}}^{z}$.

It can be shown [13] that Eqs. (3) are equivalent to the Heisenberg equations of motion

$$
\mathrm{i} \frac{\partial \sigma_{\boldsymbol{k}}^{+}}{\partial t}=2 \xi_{\boldsymbol{k}} \sigma_{\boldsymbol{k}}^{+}+g b_{\mathbf{0}} \sigma_{\boldsymbol{k}}^{z}, \quad \mathrm{i} \frac{\partial b_{\mathbf{0}}}{\partial t}=E_{\mathbf{0}} b_{\mathbf{0}}+g \sum_{\boldsymbol{k}} \sigma_{\boldsymbol{k}}^{+}
$$

averaged with respect to the wave function (2). Spin $\frac{1}{2}$ operators obey the constraint $4 \sigma_{\boldsymbol{k}}^{+} \sigma_{\boldsymbol{k}}^{-}+\left(\sigma_{\boldsymbol{k}}^{z}\right)^{2}=1$ which additionally yields

$$
\mathrm{i} \frac{\partial \sigma_{\boldsymbol{k}}^{z}}{\partial t}=2 g\left(b_{\mathbf{0}}^{\dagger} \sigma_{\boldsymbol{k}}^{+}-b_{\mathbf{0}} \sigma_{\boldsymbol{k}}^{-}\right) \text {. }
$$

This set of Eqs. (5), (6) has been previously obtained [7, 8, 15] for studying the dynamics of (1).

\section{Numerical results}

Equilibrium state of the two-component model (1) has a unique property that the condensation of molecules is simultaneously accompanied by the coherence of large Cooper pairs [10]. In the thermodynamic limit $(N \rightarrow \infty)$ it leads to the spontaneous gauge symmetry breaking. To describe this phenomenon in terms of the off-diagonal long-range order formulated by Yang [16] one introduces the following order parameters $b(t)=\left\langle b_{\mathbf{0}}\right\rangle$ and $\chi(t)=\sum_{\boldsymbol{k}}\left\langle\sigma_{\boldsymbol{k}}^{+}\right\rangle$. Mean field equations and the stationary solutions for both order parameters have been discussed in detail in Ref. [10]. As an illustration we plot in Fig. 1 the critical temperature versus the detuning parameter $2 \nu$ for the case of three-dimensional system with particle concentration $\left\langle n_{\text {tot }}\right\rangle=1$.

Starting from the stationary solution we now analyze time dependence of the order parameters in response to a sinusoidal modulation of the detuning parameter $2 \nu(t)=2 \nu_{0} \sin \omega t$. We assume that modulations occur around the Feshbach resonance in the energy window indicated by an arrow in Fig. 1. We consider frequencies $\omega$ ranging from small to large values (roughly speaking it corresponds to either the adiabatic or nonadiabatic processes). 


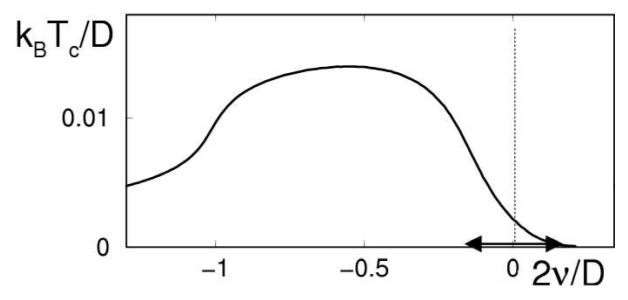

Fig. 1. The equilibrium value of the critical temperature $T_{\mathrm{c}}$ versus the detuning parameter $2 \nu$ for the three-dimensional version of the boson-fermion (BF) model (1) with a total concentration $n_{\text {tot }}=1$ and fermion band width $D$ taken as a unit for energies. In this paper we analyze the fluctuations for the detuning parameter oscillating within the regime indicated by the arrow.

Time dependent order parameters $b(t), \chi(t)$ as well as time dependent populations of the molecules and fermion atoms can be determined from Eqs. (5), (6). We solved them self-consistently using the numerical Runge-Kutta algorithm $[8,15]$. No analytical structure (like e.g. in Ref. [7]) has been imposed.
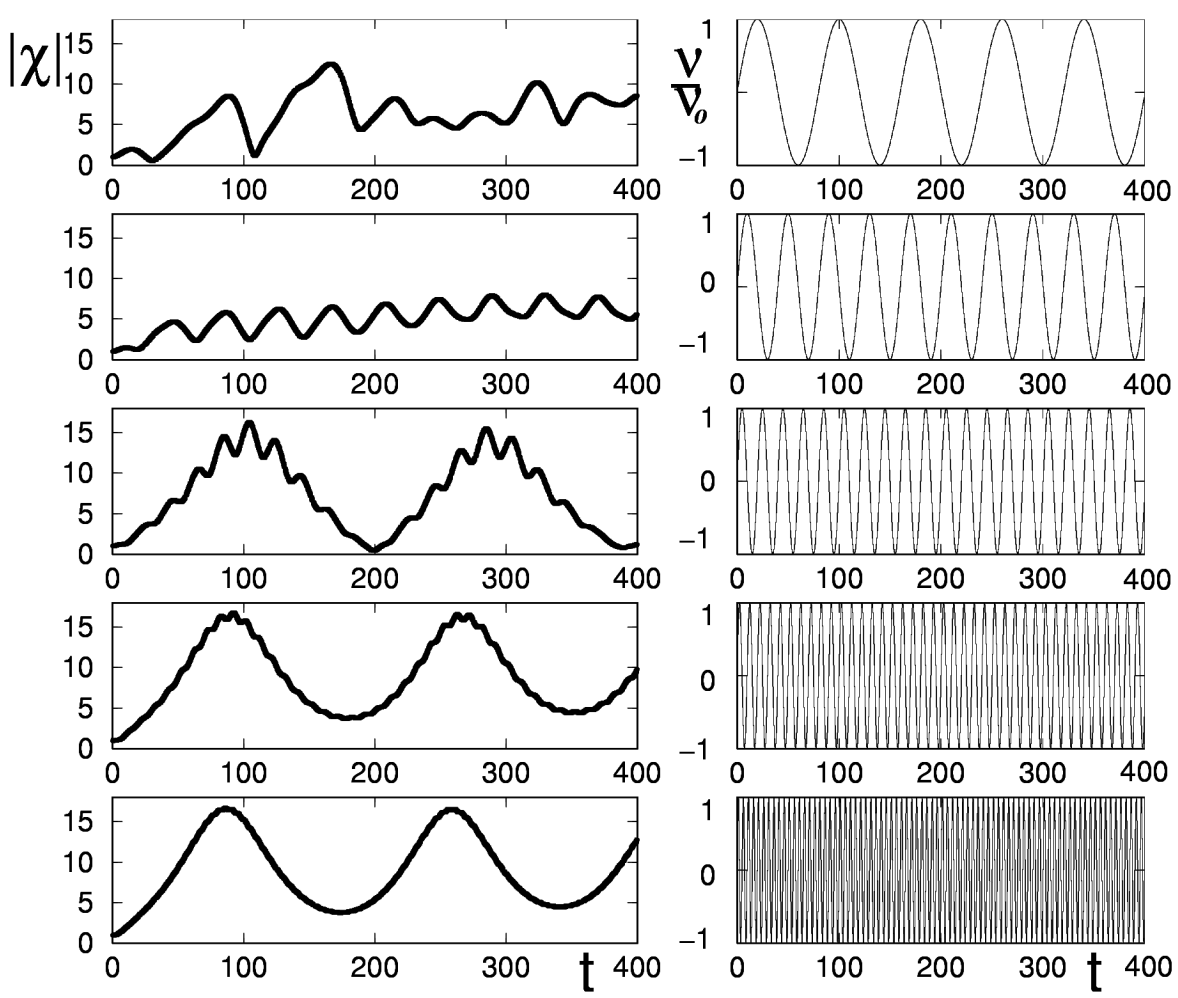

Fig. 2. Time dependence of the order parameter $|\chi(t)|$ for the detuning parameter $\nu(t)=\nu_{0} \sin 2 \pi \frac{t}{T}$, where $\nu_{0}=0.1 D$ and time $t$ is expressed in units $\hbar / D$. From the top to bottom panels in the right hand side column we show that time interval corresponds to $5,10,20,40$, and 80 oscillations of $\nu(t)$. 
For small frequencies the order parameters behave in a rather non-regular way (see the top panel in Fig. 2). Due to damping effects we observe that order parameters do not follow the modulations of $\nu(t)$. With a gradual increase in the frequency the order parameters start to vary in a kind of oscillatory way (see the bottom panels in Fig. 2). Oscillations clearly show up for frequencies $\omega \geq 2 \Delta_{0}$, where $\Delta_{0}=g\langle b(0)\rangle$ is the gap of fermion spectrum in the stationary solution. Further increase in the frequency $\omega$ smooths the envelope of the oscillations. Time dependence of the order parameter $|b(t)|$ is qualitatively similar to the behavior of $|\chi(t)|$ shown in Fig. 2.

\section{Conclusions}

We studied dynamical variation for the ground state wave function of the ultracold fermion atoms in response to time dependent detuning $\nu(t)$ modulated across the Feshbach resonance. Structure of the wave function (2) is directly related to the order parameters $\left\langle b_{\mathbf{0}}\right\rangle$ and $\left\langle\sigma_{\boldsymbol{k}}^{+}\right\rangle$. From our self-consistent treatment it turns out that: (i) the order parameters have no regular behavior in the adiabatic limit for $\omega$ being small, while (ii) they manifest quantum oscillations for sufficiently large frequencies $\omega \geq 2 \Delta_{0}$. We hope that such oscillations of the order parameters could be checked experimentally for example using the radio frequency spectroscopy [17].

\section{Acknowledgments}

This work is partly supported by the State Committee for Scientific Research under the grant No. 2P03B06225.

\section{References}

[1] M. Greiner, C.A. Regal, D.S. Jin, cond-mat/0502539.

[2] R.A. Duine, H.T.C. Stoof, Phys. Rep. 396, 115 (2004).

[3] S. Jochim, M. Bartenstein, A. Altmeyer, G. Hendl, S. Riedl, C. Chin, J. Hecker Denschlag, R. Grimm, Science 302, 2101 (2003); M.W. Zwierlein, C.A. Stan, C.H. Schunck, S.M.F. Raupach, S. Gupta, Z. Hadzibabic, W. Ketterle, Phys. Rev. Lett. 91, 250401 (2003); M. Greiner, C.A. Regal, D.S. Jin, Nature 426, 537 (2003); T. Bourdel, L. Khaykovich, J. Cubizolles, J. Zhang, F. Chevy, M. Teichmann, L. Tarruell, S.J.J.M.F. Kokkelmans, C. Salomon, Phys. Rev. Lett. 93, 050401 (2004).

[4] T. Domański, Phys. Rev. A 68, 013603 (2003).

[5] C.A. Regal, M. Greiner, D.S. Jin, Phys. Rev. Lett. 92, 040403 (2004); M.W. Zwierlein, C.A. Stan, C.H. Schunck, S.M.F. Raupach, A.J. Kerman, W. Ketterle, Phys. Rev. Lett. 92, 120403 (2004); J. Kinast, S.L. Hemmer, M.E. Gehm, A. Turlapov, J.E. Thomas, Phys. Rev. Lett. 92, 150402 (2004); C. Chin, M. Bartenstein, A. Altmeyer, S. Riedl, S. Jochim, J. Hecker Denschlag, R. Grimm, Science 305, 1128 (2004). 
[6] D.M. Eagles, Phys. Rev. 186, 456 (1969); A.J. Leggett, J. Phys. (Paris) 41, 7 (1980); S. Robaszkiewicz, R. Micnas, K.A. Chao, Phys. Rev. B 23, 1447 (1981); P. Nozieres, S. Schmitt-Rink, J. Low Temp. Phys. 59, 195 (1985).

[7] A.V. Andreev, V. Gurarie, L. Radzihovsky, Phys. Rev. Lett. 93, 130402 (2004); R.A. Barankov, L.S. Levitov, Phys. Rev. Lett. 93, 130403 (2004).

[8] M.H. Szymańska, B.D. Simons, K. Burnet, Phys. Rev. Lett. 94, 170402 (2005); M. Haque, H.T.C. Stoof, Phys. Rev. A 71, 063603 (2005).

[9] M. Holland, S.J.J.M.F. Kokkelmans, M.L. Chiofalo, R. Walser, Phys. Rev. Lett. 87, 120406 (2001).

[10] J. Ranninger, S. Robaszkiewicz, Physica B 135, 468 (1985); S. Robaszkiewicz, R. Micnas, J. Ranninger, Phys. Rev. B 36, 180 (1987); J. Ranninger, J.M. Robin, Physica C 253, 279 (1995).

[11] E. Altman, A. Vishwanath, Phys. Rev. Lett. 95, 110404 (2005).

[12] Y.M. Galperin, V.I. Kozub, B.Z. Spivak, Sov. Phys. JETP 54, 1126 (1981).

[13] W. Yi, L.-M. Duan, cond-mat/0507540.

[14] P.W. Anderson, Phys. Rev. 112, 1900 (1958).

[15] T. Domański, cond-mat/0504588.

[16] C.N. Yang, Rev. Mod. Phys. 34, 694 (1962).

[17] J. Kinnunen, M. Rodriguez, P. Törma, Science 305, 203201 (2004). 